Sonderdruck aus

\title{
Archiv der Pharmazie
}

302. Band, Heft 1, Seite 22-30

Verlag Chemie, GmbH, Weinheim/Bergstr.

W. Wiegrebe, E. Roesel, W. D. Sasse und H. Keppel

Dehydrierung des Narkotins mit dem Quecksilber(II)-acetatAeDTA-Komplex nach Knabe 
W. Wiegrebe, E. Roesel, W. D. Sasse und H. Keppel

\section{Dehydrierung des Narkotins mit dem Quecksilber(II)-acetat- AeDTA-Komplex nach Knabe *) **)}

A us dem Institut für Pharmazeutische Technologie der Technischen Universität Braunschweig u.dem Isotopenlaboratorium der Forschungsanstalt für Land wirtschaft Braunschweig.Völkenrode

(Eingegangen am 15. Mai 1968)

Bei der Dehydrierung des Narkotins (I) mit dem Quecksilber(II)-acetat-AeDTA-Komplex nach $K n a b e^{1}$ ) entstehen nur etwa $65 \%$ der berechneten Menge Quecksilber.

Wir fanden als Umsetzungsprodukte des Benzylrestes aus I Opiansäure und Mekonin. Durch Zugabe markierter Opiansäure zu einem Dehydrierungsansatz von I konnten wir nachweisen, daß Mekonin in intramolekularer Reaktion aus 3,4-Dehydro-narkotin (III) ențsteht.

\section{Dehydrogenation of Narcotine by $\mathrm{Hg}$ (II)-EDTA according to Knabe}

When narcutine (I) is dehydrogenated by the mercury(II)-a.etate-EDTA-complex according to $K n a b e^{1}$ ) only about $65 \%$ of the calculated amount of mercury is liberated. We found that meconine and opianic acid are the reaction products of the benzylic group of $I$. By addition of labelled opianic acid to a dehydrogenation-charge of $I$, we could show that meconine arises from 3,4-dehydro-narcotine (III) by intramolecular reaction.

Bei der Dehydrierung von Narkotin (I) mit dem $\mathrm{Hg}(\mathrm{JI})$-acetat-AeDTA-Komplex fand $K n a b e^{1}$ ) als basisches Spaltstück Tarkoninmethyläther(VI). Zur Einführung zweier Doppelbindungen in das Isochinolinsystem werden theoretisch $2 \mathrm{Mol} \mathrm{Hg}(\mathrm{II})$ acetat-AeDTA-Komplex (4 ,Äquivalente “) benötigt, gefunden wurden jedoch nur 2,6 Äquivalente. Diese Differenz deutet $K n a b e^{1}$ ) folgendermaßen:

Durch Einführung der ersten Doppelbindung entsteht die Imoniumverbindung II, die so instabil ist, daß sie sehr schnell hydrolytisch gespalten wird. Knabe fand Tarkoninmethyläther (VI) als basisches und formuliert Mekonin (VIII) als nichtbasisches Spaltstück. Für das von $K n a b e^{1}$ ) angestrebte Ziel, die Konstellation von I zu klären, war das Verhalten des Benzylrestes bei der Dehydrierungsreaktion ohne Bedeutung.

Bei der Dehydrierung von 9-Hydroxylaudanosin mit $\mathrm{Hg}(\mathrm{II})$-acetat-AeDTA fand $\left.K i r k^{2}\right)$ als nichtbasisches Spaltstück Veratrumaldehyd. Diesem entspricht jedoch die Opiansäure (VII) in der Oxydationsstufe, während dem Mekonin der 3,4-Di-

*) Herrn Prof. Dr. Ing., Drs. h. c. mult. Karl Winnacker zum 65. Geburtstag gewidmet.

**) 7. Mitt. über Tetrahydroisochinolyl-phenyl-carbinole;6. Mitt. Arch. Pharmaz. 296, 807 (1963)

1) J. Knabe, Arch. Pharmaz. 293, 121 (1960).

2) O. Kirk, Dissertation Braunschweig 1964, S. 30 und 39. 
methoxybenzylalkohol entspricht. Aus diesem Grunde dehydrierte Kirk seinerseits Narkotin und konnte neben Tarkoninmethyläther (VI) als einzigem basischen Spaltstück tatsächlich Opiansäure nachweisen. Durch diesen Befund wurde Knabes Erklärung ${ }^{1}$ ) für den geringen Quecksilberverbrauch in Frage gestellt.

Bei Untersuchungen an narkotinähnlichen Verbindungen beobachteten wir, daß Enamine vom Typ der 1,2-Dihydro- bzw. der 1,4-Dihydroisochinoline in der Lage sind, Veratrumaldehyd zu 3,4-Dimethoxybenzylalkohol zu reduzieren ${ }^{3}$ ). Aus diesem Grunde griffen wir die Narkotindehydrierung nochmals auf und untersuchten die Umsetzung des Benzylrestes aus I.

Auch bei unseren Versuchen verbrauchten wir durchschnittlich 2,6 Äquiv. Quecksilber. Bei der Untersuchung der nichtbasischen Produkte fanden wir nehen der von Kirk gefundenen Opiansäure (VII) das von Knabe formulierte Mekonin (VIIJ). Falls VII zu VIII reduziert wird, so muß andererseits VII auf eine noch näher zu kennzeichnende Verbindung als „Oxydationsmittel“" einwirken. Der Gedanke liegt nahe, daß hier die fehlenden 1,4, ,̈̈quivalente“" zu suchen sind: ein Mol VII entspräche $1 \mathrm{Mol} \mathrm{Hg(II)-acetat} \mathrm{(2} \mathrm{Äquiv.).} \mathrm{Nimmt} \mathrm{man} \mathrm{an,} \mathrm{daß} \mathrm{VIII} \mathrm{durch} \mathrm{Reduktion}$ von VII entsteht, so ist die gefundene Menge VIII ein Maß für die Menge VII, die ggf. als Oxydationsmittel verbraucht wird. Es mußte daher eine Methode gefunden werden, die es gestattet, VIII und VII aus der $\mathrm{Hg}$ (II)-acetat-AeDTA-haltigen Lösung zu bestimmen. Eine direkte spektralphotometrische Bestimmung ist nicht möglich, da sowohl VI als auch VII und VIII im fraglichen Bereich Absorptionsmaxima besitzen (VII $\lambda_{\max } 282 \mathrm{~nm}$; VIII $\lambda_{\max } 307 \mathrm{~nm}$; VI $\lambda_{\max } 320 \mathrm{~nm}$ ). Zu reproduzierbaren Werten gelangten wir auf folgendem Wege:

Die Reaktionslösung wird vom abgeschiedenen Quecksilber, das nach $R u p p^{4}$ ) bestimmt wurde, abfiltriert und auf $100 \mathrm{ml}$ aufgefüllt. In dieser Lösung wird das Mekonin dc abgetrennt und mit Benzol eluiert. Das Benzol wird i. Vak. abgedampft, der Rückstand in Methanol gelöst und die Extinktion bei $307 \mathrm{~nm}$ gemessen. An Hand einer Eichkurve wird der Mekoningehalt errechnet.

Zur Bestimmung der Opiansäure wird in Anlehnung an das DAB 7-DDR ${ }^{5}$ ) die Reaktionslösung mit Äthanol verdünnt und mit 3,4-Dinitrophenylhydrazinlösung nach Mühlemann ${ }^{6}$ ) versetzt. Der Niederschlag wird gewogen.

In der beschriebenen Weise wurden in 3 Dehydrierungsansätzen die Quecksilberund je 2 Mekonin-werte bestimmt. In einer 4. Dehydrierung wurde neben Quecksilber und Mekonin auch die Opiansäure bestimmt (Tab. 1).

$\left.{ }^{3}\right)$ E. Roesel, unveröffentlichte Versuche.

4) E. Rupp, Arch. Pharmaz. 243, 300 (1905).

5) DAB 7-DDR, Akademie Verlag, Berlin 1964, Band II, Monographie: Camphora.

6) Mühlemann-Bürgin, Qualitative Arzneimittelanalyse, Reinhardt-Verlag, München-Basel 1956, 2. Auflage, S. 68. 


\section{Reaktionsschema}<smiles>COc1ccc2c(c1OC)C(=O)OC2C1c2c(cc3c(c2OC)OCO3)CCN1C</smiles>

I

Weg 1<smiles>C=CC1CC[N+](C)=CCc2cc3c(c(OC)c21)OCO3</smiles>

II $\downarrow \mathrm{Oac}^{\odot}$<smiles>COc1ccc2c(c1OC)OC(O)(OC)C2CC1c2c(cc3c(c2OC)OCO3)CC[N+]1(C)C</smiles>

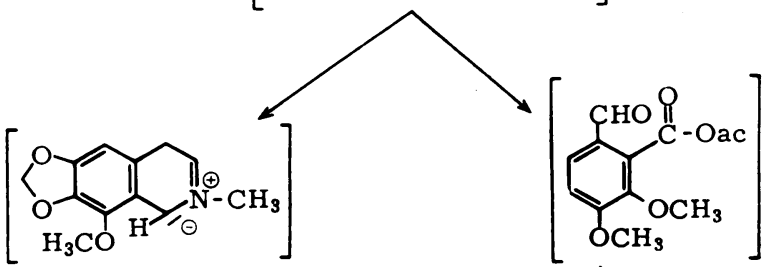<smiles>C[14CH]=O</smiles>

$\underbrace{\mathrm{COOH}}_{\mathrm{OCH}_{3}}$ VII IV

$\downarrow \mathbf{H}^{\oplus}$

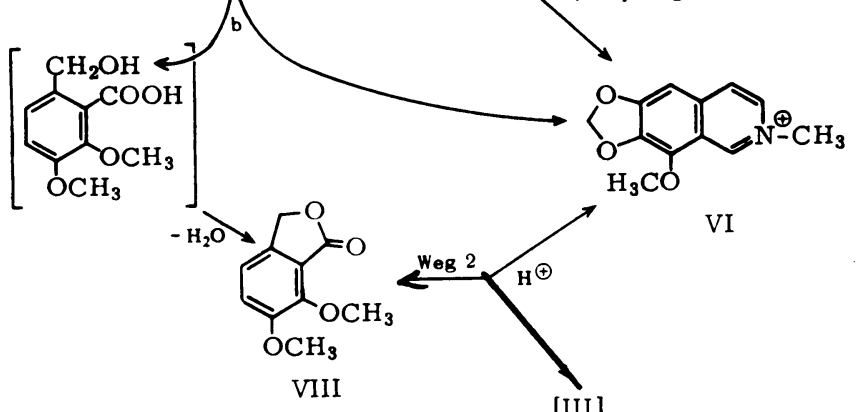

[III] 


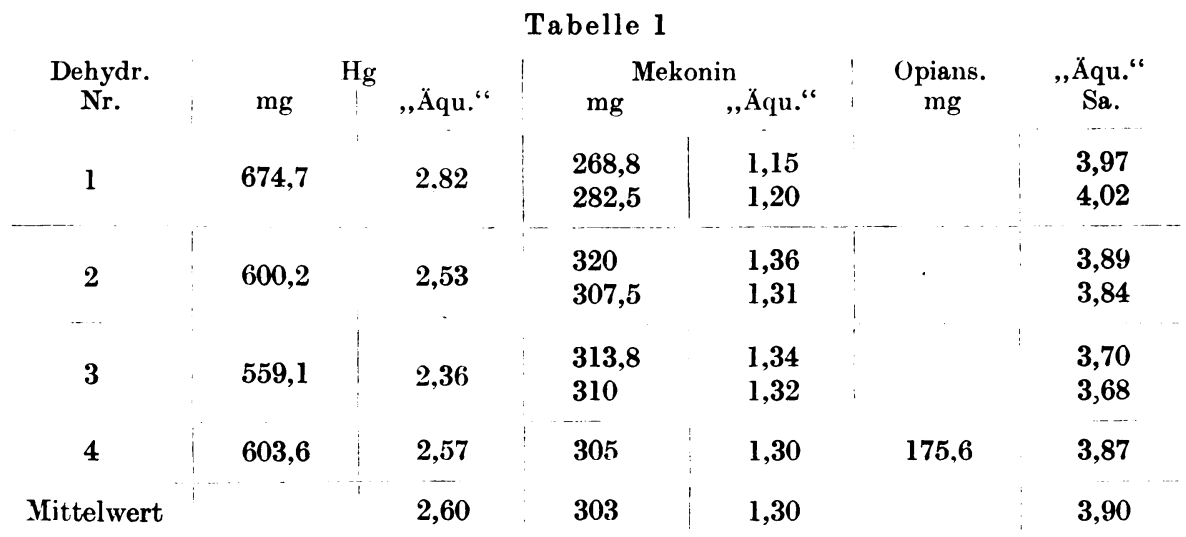

Bei einem 2,6-Äquiv. entsprechenden durchschnittlichen Verbrauch an $\mathrm{Hg}$ (II)acetat-AeDTA mußte eine 1,4-Äquivalenten entsprechende Menge Mekonin gefunden werden, das sind beim Einsatz von $1 \mathrm{~g}$ Narkotin $330 \mathrm{mg}$. Wie aus der Tabelle $\mathrm{zu}$ ersehen ist, wurden im Durchschnitt aller Bestimmungen $303 \mathrm{mg}$ Mekonin gefunden, die 1,3- Äquivalenten entsprechen. Bei der 4. Bestimmung entspricht der gefundene Wert an Opiansäure (176) gut dem theoretischen (179,8 mg).

Damit ist erwiesen, daß der geringe Quecksilberverbrauch auf das gleichzeitige Auftreten von Opiansäure und Mekonin zurückzuführen ist. Dieser Befund läßt sich folgendermaßen deuten (Reaktionsübersicht, s. vorstehend S. 24):

Unter Verbrauch von 2 Äquiv. Quecksilber geht I in die Imoniumverbindung II über ${ }^{1}$ ), die mit der 1,2-Dihydroverbindung III im Gleichgewicht steht. Eine primäre Spaltung der Molekel und anschließende Aromatisierung des 1,2,3,4-Tetrahydroisochinolinkernes kann ausgeschlossen werden: $K$ nabe $e^{7}$ ) hat 6,7-Dimethoxy-2-methyl-1,2,3,4-tetrahydroisochinolin mit dem $\mathrm{Hg}$ (II)-acetat-AeDTA-Komplex dehydriert und nur 3,4-Dihydro-6,7-dimethoxy2-methylisochinolinium-Salz erhalten.

II zerfällt unter dem Einfluß von Acetationen in VII und IV (Weg 1), das durch Aufnahme eines Protons die Imoniumverbindung $\mathrm{V}$ liefert. $\mathrm{V}$ bzw. die isomere 1,2-Dihydroverbindung (analog dem Gleichgewicht II $\rightleftarrows$ III) kann auf 2 Wegen weiterreagieren:

a) V wird durch den $\mathrm{Hg}$ (II)-acetat-AeDTA-Komplex zu VI dehydriert;

b) durch VII wird V zu VI dehydriert, wobei VII zu VIII reduziert wird.

Gegan den Reaktionsweg b) sprechen folgende Befunde:

1. Wird I in Gegenwart eines großen Uberschusses an VII mit $\mathrm{Hg}$ (II)-acetat-AeDTA dehydriert, so werden ebenfalls 2,6 Äquiv. $\mathrm{Hg}$ verbraucht, d. h. es ist kein Einfluß der freien VII auf den Verlauf der Dehydrierung zu beobachten.

2. Wird 1,2-Dihydro-2-methyl-isochinolin als Modellsubstanz in Gegenwart von VII in 1proz. Essigsäure erhitzt, so läßt sich kein VIII nachweisen; es treten allein die Disproportionierungsprodukte ${ }^{8}$ ) N-Methyl-1,2,3,4-tetrahydroisochinolin und das N-Methyl-isochinolinium-Ion neben der eingesetzten Opiansäure auf.

7) J. Knabe, Arch. Pharmaz. 292, 658 (1959).

8) O. Kirk, Dissertation Braunschweig 1964, S. 37. 
Wenn das aufgefundene VIII nicht durch die erwähnte in ter molekulare Reaktion zwischen VII und dem Imoniumion V entsteht, so muB VIII intramolekular auf folgendem Weg gebildet werden:

Die 1,2-Dihydroverbindung III, die mit II im Gleichgewicht steht, zerfällt unter dem Einfluß von Protonen in VI und VIII (Weg 2). Aus der Tatsache, daß mehr als 2 Äquiv. $\mathrm{Hg}$ verbraucht werden. und aus dem Auftreten von VII neben VIII läßt sich schließen. daß neben dem Zerfall von III (Weg 2) auch eine Dehydrierung von V durch $\mathrm{Hg}(\mathrm{II})$. acetat-AeDTA stattfindet (Weg $1 \mathrm{a}$ ). Aus der Menge des gefundenen VIII läßt sich schließen, daß die Reaktion $\mathrm{zu}^{2} / 3$ dem Weg 2 und zu $1 / 3$ dem Weg 1 folgt.

Da die vorausgegangenen Untersuchungen zur Aufklärung des Reaktionsmechanismus bei der Dehydrierung von Narkotin darauf hinweisen, daß die in tra molekulare Umsetzung von II bzw. III im Vordergrund steht, sollte durch den Zusatz ${ }^{14} \mathrm{C} \cdot \mathrm{mar}$ kierter Opiansäure zu dem Reaktionsansatz diese These gestützt werden.

Wir gingen bei der Darstellung aktiver Opiansäure von inaktiver aus, die mit Aluminiumbromid in Nitrobenzol zur 2,3-Dihydroxy-6-formyl-benzoesäure umgesetzt wurde. Nach ${ }^{9}$ ) kommt man zu sehr schlechten Ausbeuten.

An radioaktivem Material standen uns $0,1 \mathrm{mc}^{14} \mathrm{CH}_{3} \mathrm{~J}$ zur Verfügung. Das Methyljodid ${ }^{14} \mathrm{C}$ wurde mit inaktivem Methyljodid verdünnt und zu der methanolischen Lösung der Dihydroxysäure gegeben. Eine Methylierung der Dihydroxysäure auf diesem Wege war, wie wir aus Vorversuchen wußten, zu erreichen. Allerdings entsteht bei dieser Umsetzung kein einheitliches Produkt. Als wahrscheinlich ist ein Gemisch aus 2-Hydroxy-3-methoxy-6-formylbenzoesäure, 2-Methoxy-3-hydroxy6-formylbenzoesäure, Opiansäure und Ausgangsmaterial (Dihydroxysäure) anzunehmen.

Im Rahmen unserer Untersuchung ist die genaue Lokalisierung der Aktivität im Opiansäure-Molekül ohne Bedeutung ; es war aber wesentlich, daß die Dihydroxysäure an einer Phenolgruppierung mit ${ }^{14} \mathrm{CH}_{3} \mathrm{~J}$ methyliert worden war. Das Säurengemisch wurde anschließend mit Diazomethan vollständig methyliert und der methoxylmarkierte Opiansäuremethylester mit Salzsäure zu VII verseift.

Messungen im TRI-CARB-Flüssigkeits-Szintillationsspektrometer (Fa. Packard, Mod. $314 \mathrm{X}$ ) ergaben die Impulsrate für $10,6 \mathrm{mg}$ Opiansäure-methoxy ${ }^{11} \mathrm{C}$ von $1.621 \mathrm{Ipm}$. Bei einer Zählausbeute des Gerätes von $70,1 \%$ für ${ }^{14} \mathrm{C}$ und einem Nulleffekt der Szintillationslösung von $48 \mathrm{Ipm}$ ergab sich eine Zerfallsrate von $2.143 \mathrm{Zpm}$ oder von $42,860 \mathrm{Zpm} / \mathrm{mM}$ Opiansäure. Die niedrige Einbaurate führen wir auf Verluste bei der Zugabe und auf Hydrolyse des Methyljodids zurück. Angegeben sind die Mittelwerte aus 3 Messungen während 10 Min. je Probe.

Bereitung der Szintillationslösung:

$40 \mathrm{mg}$ 1,4-Bis(5-phenyloxazolyl)-benzol, 5,6 g 2,5-Diphenyloxazol, $40 \mathrm{~g}$ Naphthalin, $800 \mathrm{ml}$ Dioxan.

Nun wurde Narkotin unter den beschriebenen Reaktionsbedingungen mit Hg(II)acetat-AeD'TA und dem Zusatz eines dreimolaren t'berschusses an ${ }^{14} \mathrm{C}$-Opiansäure

9) Beilsteins Handbuch der (Organischen Chemie, 4. Aufl., Band X, S. 990. 
dehydriert. Es wurden wie bei vorangegangenen Untersuchungen wieder 2,6 Äquiv. $\mathrm{Hg}$ verbraucht.

Wäre nur Reaktionsweg $1 \mathrm{~b}$ durchlaufen worden, so wäre aus I unmarkierte Opiansäure entstanden, die V zu VI dehydriert hätte, wobei sie selbst in VIII übergegangen wäre. Da eine $3 \mathrm{Mol}$ entsprechende Menge markierte Opiansäure dem Dehydrierungsansatz zugesetzt wurde, müßten sich unmarkierte Opiansäure zu markierter wie $1: 4$ verhalten, d. h. das entstandene Mekonin sollte $75 \%$ der molaren Einbaurate von VII aufweisen. Wir isolierten das Mekonin durch präparative Schichtchromatographie und untersuchten es im Flüssigkeitszintillationsspektrometer.

Für $10 \mathrm{mg}$ Mekonin in $20 \mathrm{ml}$ Dioxan-Szintillator ergab sich eine Impulsrate von $28 \mathrm{Ipm}$. Die Aktivität der Probe ist somit innerhalb der Fehlergrenzen gleich dem Nullwert der Szintillatorlösung (32 Ipm/20 ml).

Die Messung zeigt, daß das bei der Dehydrierung entstandene Mekonin ausschließlich dem Narkotin entstammt, denn es ist inaktiv. Bei der quantitativen Bestimmung wurde die 1,33 Äquiv. entsprechende Menge Mekonin gefunden. Die eingesetzte markierte Opiansäure konnte in vollem Umfange wie beschrieben zurück gewonnen werden.

Damit ist der Nachweis erbracht, daß Mekonin VIII nur durch intramolekulare Reaktion (Weg 2) entsteht. Eine Reduktion freier Opiansäure (Weg 1 b) durch das Enamin V findet nicht statt.

\section{Beschreibung der Versuche}

Dehydrierung von Narkotin mit $\mathrm{Hg}$ (II)-acetat-AeDTA in 1 proz. Essigsäure und Bestimmung des Quecksilbers

$\mathrm{Zu}$ der warmen Lösung von 3,08 g Hg(II)-acetat (8 Äquiv.) und 3,6 g AeDTA in $30 \mathrm{ml}$ 1proz. Essigsäure wurde die Lösung von $1 \mathrm{~g}$ Narkotin in $2 \mathrm{ml} 2 \mathrm{n} \mathrm{HCl}$ und $25 \mathrm{ml} 1$ proz. Essigsäure gegeben. Das Kölbchen wurde zweimal mit $5 \mathrm{ml}$ lproz. Essigsäure nachgespült und die Mischung $11 / 2$ Std. auf dem siedenden Wasserbade erwärmt. Nach dem Erkalten wurde das Quecksilber abgetrennt, noch einige Male mit insgesamt $20 \mathrm{ml}$ lproz. Essigsäure nachgewaschen und nach ${ }^{4}$ ) bestimmt (s. Tab. 1).

Quantitative Bestimmung von Mekonin

Die vom Quecksilber abgetrennte Reaktionslösung wurde in ein Meßkölbchen filtriert und $100 \mathrm{ml}$ aufgefüllt. $0,4 \mathrm{ml}$ dieser Lösung wurden bandförmig auf eine DC-Platte aufgetragen (Schicht: Kieselgel $\mathbf{P F}_{254}$ Merck; Schichtdicke: $0,2 \mathrm{~mm}$; bei Raumtemperatur getrocknet, über Calciumchlorid aufbewahrt) und zweimal $15 \mathrm{~cm}$ mit einer Mischung aus Benzol und Methanol (9:1) entwickelt. Das unter dem UV-Licht markierte Band wurde abgehoben; eine gleichgroße Fläche wurde als Blindwert abgehoben und wie der Hauptwert behandelt.

Das Kieselgel wurde mit einigen Tropfen Wasser desaktiviert und 10 Min. mit $10 \mathrm{ml}$ Benzol unter Rückfluß gekocht. Durch eine Fritte 3 G 5 M wurde vom Kieselgel abfiltriert. Kolben und Fritte wurden mit $10 \mathrm{ml}$ Benzol nachgespült. Das Benzol wurde i. Vak. abdestilliert und der Rückstand mit Methanol zu $25 \mathrm{ml}$ gelöst. Bei $307 \mathrm{~nm}$ wurde die Extinktion gegen den Blindwert gemessen und an einer Eichkurve der Mekoningehalt abgelesen (s. Tab. 1). 
Aufstellung der Mekonin-Eichkurve und Kontrolle der Bestimmungsmethode

Die Eichkurve soll den Extinktionsbereich von $\mathrm{E}=0,1$ bis $\mathrm{E}=1,1$ erfassen. Aus der molaren,Extinktion $\log \varepsilon=3,53$ bei $307 \mathrm{~nm}$ errechnet sich für die Konzentration 1,5 mg Mekonin in $25 \mathrm{ml}$ Methanol eine Extinktion von $\mathrm{E}=1,05$.

Verschiedene Einwaagen wurden direkt in Methanol gelöst, die Extinktionen gemessen und ihre Werte in ein Koordinatensystem eingetragen, bei dem auf der Abszisse mg Mekonin in $25 \mathrm{ml}$ abgetragen sind, auf der Ordinate die Extinktion E. Im Bereich von $0,25 \mathrm{mg}-1,5 \mathrm{mg}$ Mekonin/25 ml Methanol verläuft die Kurve linear.

Zur Kontrolle der Bestimmungsmethode wurden 74,9 mg Mekonin in $10 \mathrm{ml}$ Chloroform gelöst, verschiedene Vol. auf Platten aufgetragen und in der oben beschriebenen Weise behandelt. Es zeigte sich, daB die Methode reproduzierbare Ergebnisse liefert.

\begin{tabular}{c|c|c|c|c}
$\begin{array}{c}\mathrm{ml} \\
\text { aufgetragen }\end{array}$ & $\begin{array}{c}\text { mg/25 ml } \\
\text { Sollwert }\end{array}$ & $\mathrm{E}$ & $\begin{array}{c}\mathrm{mg} / 25 \mathrm{ml} \\
\text { Istwert }\end{array}$ & $\begin{array}{c}\% \\
\text { des Sollwertes }\end{array}$ \\
\hline 0 & 0 & 0,008 & - & - \\
0,06 & 0,45 & 0,34 & 0,46 & 102 \\
0,1 & 0,75 & 0,558 & 0,75 & 100 \\
0,16 & 1,2 & 0,84 & 1,15 & 96 \\
0,2 & 1,5 & 1,08 & 1,475 & 98,5
\end{tabular}

Quantitative Bestimmung von Opiansäure ${ }^{5}$ )

$20 \mathrm{ml}$ der auf $100 \mathrm{ml}$ aufgefüllten Reaktionslösung wurden mit $30 \mathrm{ml}$ Äthanol und $40 \mathrm{ml}$ 3,4-Dinitrophenylhydrazinlösung ${ }^{6}$ ) versetzt und 5 Min. auf dem Wasserbade erwärmt. Nach dem Abkühlen wurde 1 Std. im Kühlschrank aufbewahrt. Der Niederschlag wurde auf einer Fritte $1 \mathrm{G4}$ gesammelt, mit Wasser gewaschen, $1 / 2$ Std. bei $120^{\circ}$ getrocknet und nach 1 Std. gewogen.

$1 \mathrm{mg}$ VII-Dinitrophenylhydrazon = 0,5385 mg VII. Gef.: 65,2 mg VII-Dinitrophenylhydrazon $=35,1 \mathrm{mg}$ VII in $20 \mathrm{ml}$ Reaktionslösung. Schmp. $275^{\circ}$ (Koflermikroheiztisch) $\left.\left[269-270^{\circ}{ }^{10}\right)\right]$.

Behandlung von 1,2-Dihydro-2-methyl-isochinolin mit Opiansäure in 2.proz. Essigsäure

Nach ${ }^{11}$ ) wurde 1,2-Dihydro-2-methyl-isochinolin hergestellt. Unter Stickstoffbegasung wurden $475 \mathrm{mg}$ 1,2-Dihydrobase $(0,0033 \mathrm{Mol})$ mit einer Lösung von $725 \mathrm{mg}$ VII $(0,0033 \mathrm{Mol}$ $+5 \%$ tberschuB) in $50 \mathrm{ml} 2$ proz. Essigsäure übergossen. Die Mischung wurde 2 Std. auf dem Wasserbade erwärmt. Nach dem Erkalten wurde mit Āther ausgeschüttelt, dann wurde mit Ammoniaklösung auf pH 8,5 eingestellt und mit Äther ausgeschüttelt. Nach dem Trocknen und Einengen der Åtherphasen wurde chromatographiert.

In der sauren Ausschüttelung ist nur VII nachzuweisen, in der alkalischen 1,2,3,4-Tetrahydroisochinolin. Zum Vergleich lief authentisches Material mit.

DC: Sorptionssahicht: Kieselgel HF $_{254}$ Merck, Fließmittel: Benzol $9+$ Methanol 1. Laufstrecke: $10 \mathrm{~cm}$. Detektion: UV-Licht $254 \mathrm{~nm}$, Formalin - Schwefelsäure, 30 Min. auf $120^{\circ}$ erwärmen, Dragendorff's Reagens.

10) O. Kirk, Dissertation, Braunschweig 1964, S. 81.

11) H. Schmid, P. Karrer, Helv. chim. Acta 32, 960 (1949). 


\begin{tabular}{c|c|c|c} 
& Rf & Dragendorff & Formalin $-\mathrm{H}_{\mathbf{2}} \mathrm{SO}_{4}$ \\
\hline $\begin{array}{c}\text { VII } \\
\text { VIII }\end{array}$ & am Start & $\begin{array}{l}\text { sehr schwach } \\
\text { schwach }\end{array}$ & $\begin{array}{l}\text { gelbbraun } \\
\text { blauviolett }\end{array}$ \\
$\begin{array}{l}\text { N-Methyl-1,2,3,4- } \\
\text { tetrahydroisochinolin }\end{array}$ & 0,7 & + & -
\end{tabular}

Darstellung von ${ }^{14} \mathrm{C}$-markierter Opiansäure

a) Darstellung von 2,3-Dihydroxy-6-formylbenzoesäure

$100 \mathrm{~g}$ Aluminiumbromid wurden in $300 \mathrm{ml}$ Nitrobenzol gelöst, die Lösung wurde auf dem Wasserbad auf $55^{\circ}$ erwärmt. Dann wuirden $8,0 \mathrm{~g}$ VII hinzugefügt, und das Gemisch wurde weitere $8 \mathrm{Std}$. auf $55^{\circ}$ erwärmt. Die erkaltete Lösung wurde auf $300 \mathrm{~g}$ Eis gegossen. Es bildeten sich 2 Phasen. Die wäBrige Phase wurde stark angesäuert und mit Äther so lange ausgeschüttelt, bis die Reaktion mit Eisen(III)-chloridlösung negativ verlief. Der Äther wurde über Natriumsulfat getrocknet und i. Vak. abdestilliert. Der Rückstand, ein braunes Ol, erstarrte bald. Das Kristallisat wurde aus heißem Wasser unter Zusatz von Kohle mehrfach umkristallisiert. Ausbeute: $3,4 \mathrm{~g}=49,1 \%$ d. Th., Schmp. $171-173^{\circ}$.

b) Methylierung der 2,3-Dihydroxy-6-formylbenzoesäure

$2,0 \mathrm{~g}$ Dihydroxysäure wurden in $25 \mathrm{ml}$ Methanol gelöst und mit $\mathrm{n} \mathrm{NaOH}$ auf pH 9 eingestellt. Nun wurde 0,1 mc ${ }^{14} \mathrm{C}$-Methyljodid aus einer Ampulle mit $1 \mathrm{ml}$ Methyljodid in die Lösung gespült. Es wurde auf dem Wasserbad unter Rühren und Stickstoffbegasung 3 Std. im Sieden gehalten. Nach jeder Std. wurde $1 \mathrm{ml}$ Methyljodid hinzugefügt. Nach dem Abkühlen wurden Methanol und überschüssiges Methyljodid i. Vak. abgezogen, der Rückstand in Wasser aufgenommen, angesäuert und mit Äther extrahiert. Nach dem Trocknen des Âthers über Natriumsulfat wurde die Lösung bis zur Trockne eingeengt. Zurück blieb ein hellbraunes Kristallisat, welches aus Wasser umkristallisiert wurde.

c) Umsetzung des bei b) erhaltenen Säurengemisches mit Diazomethan

Das bei b) erhaltene Produkt wurde in wenig Methanol gelöst, mit der ätherischen Lösung von Diazomethan aus 4,0.g Nitrosomethylharnstoff versetzt und 24 Std. stehengelassen. Nach dem Abziehen des überschüssigen Diazomethans und der Lösungsmittel blieb ein gelbes Ol zurück, das im DC (Fließmittel: Benzol 8, Methanol 2) den gleichen Rf-Wert wie authentischer Opiansäuremethylester aufweist.

d) Umsetzung des markierten Opiansäuremethylesters mit 10proz. Salzsäure

Der ölige Rückstand von c) wurde in 10proz. Salzsäure aufgenommen und die Lösung während $3 \mathrm{Std}$. in mäßigem Sieden gehalten. Nach dem Abkühlen wurde mit Äther ausgeschüttelt. Die ätherische Lösung wurde mit 5proz. Natriumhydrogencarbonatlösung ausgeschüttelt und diese nach dem Ansäuern wieder mit Äther extrahiert. Der Äther wurde über Natriumsulfat getrocknet und bis auf wenige $\mathrm{ml}$ abdestilliert. Die ${ }^{14} \mathrm{C}$-markierte Opiansa kristallisierte bald aus. Schmp. 148-149 ${ }^{\circ}$. Der Mischschmp. mit authentischer Substanz èrgab keine Depression. Ausbeute: $200 \mathrm{mg}$.

Dehydrierung von Narkotin mit $\mathrm{Hg}(\mathrm{II})$-acetat-AeDTA in Gegenwart von ${ }^{14}$ C-markierter Opiansäure

$0,1 \mathrm{~g} \mathrm{I}$ und $0,153 \mathrm{~g}{ }^{1 \Perp} \mathrm{C}$-markierte VII wurden in $3 \mathrm{ml}$ 1proz. Essigsäure und einigen Tropfen $2 \mathrm{n} \mathrm{HCl}$ in der Wärme gelöst und zu der warmen Lösung von 0,36 g AeDTA und $0,31 \mathrm{~g} \mathrm{Hg}$ (II)-acetat in $3 \mathrm{ml}$ lproz. Essigsäure gegeben. Das Gemisch wurde $11 / 2 \mathrm{Std}$. auf dem Wasserbade erwärmt. Die Bestimmungen des Quecksilbers, des Mekonins und der Opiansäure wurden wie bereits beschrieben ausgeführt. Es ergaben sich: 
2,62 Åquiv. Hg + 1,33 Åquiv. (aus dem gef. Mekonin ber.) = 3,95 Äquiv.

Die Isolierung des Mekonins gelang durch präp. Schichtchromatographie. Kieselgel $\mathrm{PF}_{254}$ Merck; Platten mit $2 \mathrm{~mm}$ Schichtdicke, Laufstrecke $15 \mathrm{~cm}$, Fließmittel : Benzol 9 + Methanol 1. Das unter dem UV-Licht $254 \mathrm{~nm}$ markierte Band wurde abgehoben und weiter wie bei der Mekoninbestimmung behandelt. Mekonin wurde so kristallin erhalten. Schmp. $101^{\circ}$. Der Mischschmp. mit authentischer Substanz ergab keine Depression.

\section{Allgemeine Angaben}

Schmp. sind unkorrigiert angegeben. UV-Spektren: Gerät PMQ II der Fa. Zeiss.

Herrn Prof. Dr. Awe † sind wir für Diskussionen unserer Ergebnisse dankbar; dem Land Niedersachsen sind wir für die großzügige Förderung dieser Arbeit zu Dank verpflichtet.

Anschriften: Doz. Dr. W. Wiegrebe, 33 Braunschweig, Pockelsstr. 4. Dipl.-Chem. H. Keppel, Isotopenlaboratorium der Forschungsanstalt fur Landwirtschaft, 33 Braunschweig, Bundesallee 50.

[Ph 593]

(C) Verlag Chemie GmbH, Weinheim/Bergstr., 1969

Bel der Wiedergabe von Gebrauchsnamen, Handelsnamen, Warenbezeichnungen u.dgl. handelt es sich hăufg um gesetzlich geschützte eingetragene Warenzeichen, auch wenn sie nicht als solche mit (B) gekennzeichnet sind. 